\title{
Reliability Assessment of Power System Backup Protection in Smart Grid Control Center Using Phasor Measurement Units (PMU)
}

\author{
A.R.Motavalian ${ }^{1}$, N.Moadabi ${ }^{1}$ and G. B. Gharehpetian ${ }^{1}$ \\ ${ }^{1}$ Electrical Engineering Department \\ Amirkabir University of Technology, Tehran. Iran \\ Phone/Fax number: +0098 216454 3341, e-mail: Motevalian_a@aut.ac.ir, Moadabi_N@aut.ac.ir, grptian@aut.ac.ir
}

\begin{abstract}
Catastrophic failures in power grid due to maloperation of traditional backup protection system (e.g. third zone of distance relays) was one of the main causes of many cascade trips. This paper assess a new wide-area backup protection system (WABPS) based on phasor measurement units (PMU), to improve third zone of distance relays to avoid its mal-operation. Based on this assessment, phasor measurement units with high speed communication system such as fiber optic and using time transfer with GPS system have dynamic adjustment for reliable backup protection system to avoid mal-operation. The new backup protection system is based on phasor measurement units (PMU) obtained from studies on the IEEE 9 bus test system using Matlab Simulink. It can successfully distinguish between actual and virtual faults for interconnected lines
\end{abstract}

\section{Key words}

Smart Grid, Phasor management unit (PMU), Backup Protection, Third zone of Distance Relay, Wide-area monitoring, protection, control system (WAMPCS), Wide-area Backup protection system (WABPS)

\section{Introduction}

$\mathrm{N}$ owadays, electrical grids are more complex due to their fast development and considering distributed generation (DG) sources, electricity market restructuring, and deregulation, and the existence of independent power producers (IPP). Therefore, availability of electric power with high reliability and adequate power quality are essential for electrical networks. In this regard, new technologies such as smart grids has introduced a new standard for monitoring, protection, and control systems in order to increase the safety of grids, providing reliable electricity power, reduce the unwanted blackouts, rapid response to changes in the electrical system conditions, fault detection and recovery of electrical grids. Here, Wide-Area Monitoring, Protection and Control Systems (WAMPCS) are vital for Electrical Management Systems (EMS) [1].

Generally, collapse of the power system is done in four stages as follows [2].

- $\quad$ First stage (simple fault): the system is designed based on $\mathrm{N}-1$ constraint criteria and expected to work continuously with the first fault. If the next fault or two synchronic events occur, the system is converted to $\mathrm{N}-2$. This secondary fault may be caused by protective relays, human fault or incorrect analysis of the power system.

- Second stage (load encroachment): secondary fault may be caused an overload in the system and need to trip in the overload line, therefore the active power outages from overloaded lines to be transferred to other lines.

- $\quad$ Third stage (power swing): active power swing occurrence in power system causes the third zone mal-operation of distance relay.

- Fourth stage (islanding): with spreading lines outage of the power system, practically there will be an island mode which can be lead to voltage collapse in power system.

Mal-operation of distance protection third zone in circumstances like load encroachment, power swing and voltage instability may be lead to the spread wide-area blackouts. NERC presented 14 recommendations to prevent widespread blackouts in Final Report on the August $14^{\text {th }}, 2003$ Blackout in the United States and Canada[3].According to this report, using synchronization time criteria in protective system to improve performance, particularly in the third zone of distance relay is necessary.

The paper is organized as follows. In section II, maloperation of third zone of distance relay is reviewed. Then, in Section III by means of phasor management unit, a new Wide-Area backup protection system (WABPS) is suggested to be employed in WAPS. In Section IV, the performance of proposed protection system is simulated on the IEEE 9 bus test system. Conclusions of this paper are given in Section V.

\section{Mal-Operation of the Third Zone}

Generally, the first and second zones are considered as main protection of the transmission lines whereas the third zone is known as the backup protection. The first zone covers about 80 to 85 percent of the line without any time delay. This zone is used as the main protection. Second zone covers the entire length of the line plus 25 to 50 percent of the next shortest line and operation time is set about 0.25 to 0.4 seconds. Third zone covers the entire area protected by the first and second zones plus 25 percent of the next long line (third line) and delay time is 
set between 0.6 to 1.5 seconds. This zone is used for backup protection.

In this section, the causes of mal-operation of third zone will be discussed. As can be seen in the Fig (1), a classic layout of the third zone of distance relay is displayed. This structure serves to protect each line separately. Hence, mal-operation of protection system in abnormal condition is very high.

\section{A. LOAD ENCROACHMENT AND POWER SwING}

In swing conditions, amplitude and phase angle of voltage and current oscillate, which may caused maloperation particularly in the third zone of distance relay. Distance protection relays are equipped with power swing detection modules,e.g. Concentric characteristics method. Concentric Characteristic method is the simplest way to measure speed of impedance oscillations. It monitors the impedance trajectory between two concentric areas which is defined in the protection relays. The advantage of this method is that the power swing is detected before impedance entrance into protection zones. One of the limitations of this method is that the concentric characteristics are barriers for outer characteristics to extend the load area. These factors lead to serious restrictions for the long lines and heavy loads [4].

Blinder Schemes is based on measuring the travelling time of the impedance trajectory passing a blinder zone. It can be used independently of the distance zones characteristics and load impedance can be placed inside a blinding scheme areas. The disadvantage of this method is the complex coordination and regulation of the blind scheme, according to network conditions [4].

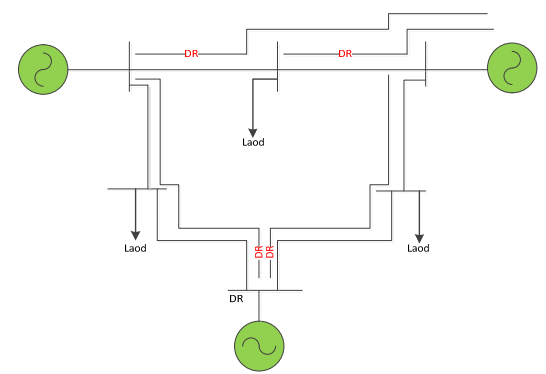

Fig1. Classic layout of the third zone of distance relay

\section{B. Voltage InStabiLITy}

The main cause of voltage instability is shortage of reactive power in power system [5]. Mal-operation of distance relay in terms of voltage instability can occur by the third zone relay which having a maximum area of protection as backup protection [6]. The worst case of voltage instability occurs when the dynamic load tries beyond the capability of transmission line or generation to be used [7]. Various methods for fault detection from voltage instability are presented. Using derivative of voltage and calculation the voltage changes are not always responsive, as step-voltage increase due to load shedding or switching of capacitors. When the network is still faulty, derivative of voltage near the voltage collapse is large and negative [8], [9], [10], and [11]. Using power estimate through transmission line at any moment and blocking the third zone performance is another way to distinguish between fault and voltage instability [12], [13], [14]. Using line voltage stability is another method that can be used for detecting fault and voltage instability [15]. The disadvantage of this method is the dependence and connection between substations. If connection between the substations to be cut, fault detection is not performed properly.

In the aforementioned solutions, two aspects are considered:

- Discovered the power swing or voltage instability relay for the blocking the third zone

- Calculated fault location and permission to the third zone relay

Therefore, this paper attempts to evaluate the feasibility and assessment new backup protection systems to reliable and improve the current backup protection.

\section{The Proposed Structure}

In the past, due to the mal-operation of third zone, some references suggest removing this backup protection and in other ones, the necessity of this backup protection is mentioned [16].

Wide-area monitoring, protection and control system (WAMPCS) collect information through fast and reliable communication to deal with large disturbances. Here, synchronized measurement technology (SMT) plays very important role [1].

Real time measurement of the wide-area protection system (WAPS) will be so useful for implementing adaptive protection system especially in back up applications.

On the other hand, sending information of phasor measurement units from different parts of the grid to main control center is associated with a delay of several tens of milliseconds. Therefore, development of wide-area protection system (WAPS) using phasor measurement units (PMU) cannot be so enough for main protection system.

In the proposed structure, the information collected for data processing is sent to main control centers and after processing stage, appropriate control and protection commands are sent to local equipments. Fig. (2) Shows the structure of the proposed backup protection system.

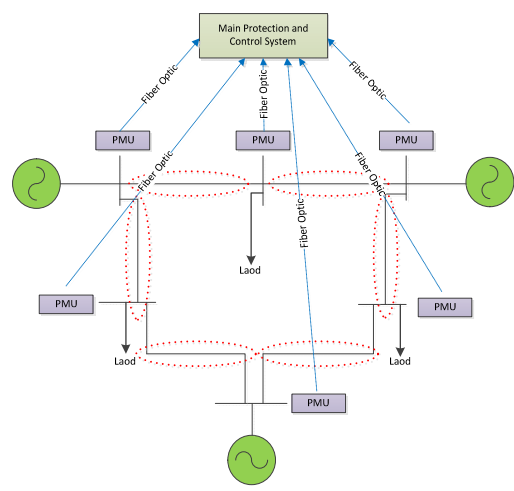

Fig2. Wide-Area Backup Protection System (WABPS) Proposed 
PMUs measure positive sequence (and negative and zero sequence quantities, if needed) voltages and currents of a power system in real time with precise time synchronization. This common time reference is independent of the geographical position of the measuring device. All measurements are done with the GPS one pulse per second (1pps) [17].

The result of the measurement is a vector $\mathrm{X}$ (synchronized phasor) as given below:

$$
\begin{aligned}
& x(t)=x r+j x i=\left(\frac{x m}{\sqrt{2}}\right) e^{j \varphi}= \\
& =\frac{x m}{\sqrt{2}}(\operatorname{Cos} \varphi+j \operatorname{Sin} \varphi)
\end{aligned}
$$

Where $\mathrm{xm}$ is the amplitude of the filtered synchronized vector and $\theta$ is the phase angle relative to a cosine function at nominal frequency. IEEE C37.118 [17] specifies that the angle $\theta$ is 0 degrees when the maximum of the signal to be measured coincides with the GPS pulse and -90 degrees if the positive zero crossing coincides with the GPS pulse.

The most commonly used method of calculating phasors from sampled data is that of Discrete Fourier Transform (DFT). The sampling clocks are usually kept at a constant frequency even though the input signal frequency may vary by a small amount around its nominal value [18].

Communication links used by WAPS include wired and wireless options [18]. Communication channel capacity is not often a limiting factor for transmission, limiting factor is latency in the data path, that in some applications of phasor measurement units (PMU), this delay causes a general delay in information transfer measured in the process. The communication options for WAPS include the following [19]:

- Twisted pair

- Fiber optic

- Microwave

- $\quad$ Power Line Carrier (PLC)

- Satellites

Delays due to the use of PMUs and the communication link involved are due primarily to the following reasons, Transducer delays, and Window size of the DFT, Processing time, and Data size of the PMU output, Multiplexing, and transitions, Communication link involved and Data. Delay calculations form an important aspect of WAPS; Communication delay can be expressed as [20]:

$$
\tau=\tau \mathrm{f}+\tau \mathrm{p}+\frac{\mathrm{L}}{\mathrm{R}}+\theta
$$

Where $\tau$ is the total link delay, $\tau f$ is the fixed delay associated with transducers used, DFT processing, data concentration and multiplexing, $\tau p$ is the link propagation delay, $\mathrm{L}$ is the amount of data transmitted, $\mathrm{R}$ is the data rate of the link, and $\theta$ is the associated random delay jitter. In applications where phasor measurement units (PMU) are involved with protection system or dynamics study, latency will be so important. The optical fiber can be installed along the cable or transmission line. Optical fiber has more bandwidth than copper wires. Table (1) indicates the delays of various communication links when using PMUs in a WAPS.

Table1. Delay calculations associated with various communication links [20]

\begin{tabular}{|c|c|}
\hline Communication Link & Associated Delay One Way $(\mathrm{ms})$ \\
\hline Fiber - Optic Cables & $\sim 100-150$ \\
\hline Digital MicroWave Links & $\sim 100-150$ \\
\hline Power Line (PLC) & $\sim 150-350$ \\
\hline Telephone Lines & $\sim 200-300$ \\
\hline Satellite Lines & $\sim 500-700$ \\
\hline
\end{tabular}

An architecture involving PMUs, communication links, and data concentrators must exist in order to realize the full benefit of the PMU measurement system. Phasor data concentrators (PDCs) is a kind of data concentrators to gather phasor data from several PMUs, reject bad data, align the time-stamps, discrete event and create a coherent record of simultaneously recorded data from a wider part of the power system.

According to the flow chart presented in Fig. (3), each PMU calculates the voltage and current phasors and sends them to the protection and control center through appropriate communication links, such as optical fiber. In the control center, $\mathrm{Rf}$ is calculated from equation (6).

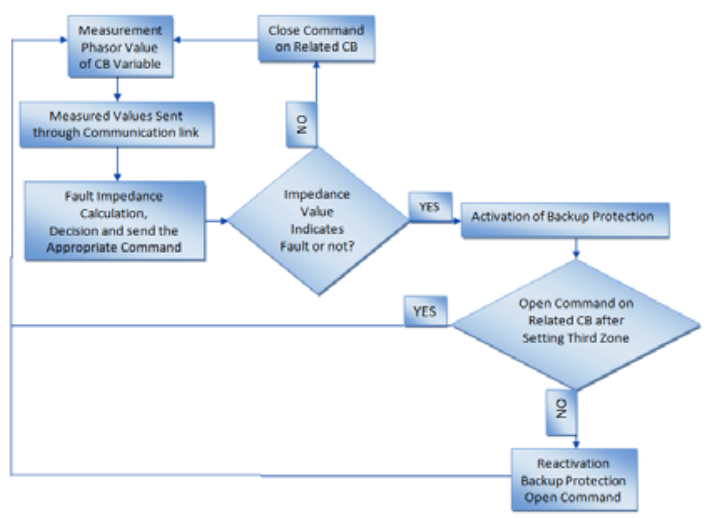

Fig3.Flow Chart of Proposed Structure

Here, to distinguish between real fault (Short circuit) and virtual fault (Load Encroachment), Rf is calculated and processed. This parameter is based on the measured resistance value ( $\mathrm{Rf}$ ) between the buses. At the beginning of this calculation, resistance value (Rf) is varies from zero to several $M \Omega$. Fig. (4) shows the method for $R f$ calculation. If value of the calculated resistance keeps very high (approximate in Mega Ohms) indicate that system is in normal operating mode.

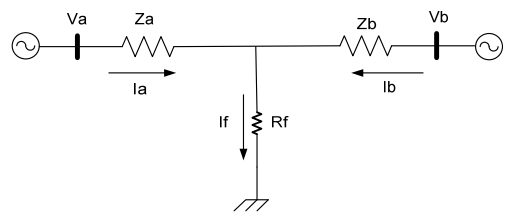

Fig4. The calculation of fault resistance $\left(\mathrm{R}_{\mathrm{f}}\right)$ [21]

$$
V a=I a Z a+I f R f \rightarrow \frac{V a}{I a}=Z a+\frac{I f}{I a} R f
$$




$$
V b=I b Z b+I f R f \rightarrow \frac{V b}{I b}=Z b+\frac{I f}{I b} R f
$$

From equations (3) and (4) we get:

$$
\frac{V a}{I a}+\frac{V b}{I b}=(Z a+Z b)+\left(\frac{I f^{2}}{I a \cdot I b}\right) R f
$$

Since we have:

$(Z a+Z b)=Z_{T L}$

$(I a+I b)=I_{f}$

Then we have:

$$
R f=\left(\frac{V a}{I a}+\frac{V b}{I b}-Z_{T L}\right) \cdot\left(\frac{I a I b}{I f^{2}}\right)
$$

The value of $\mathrm{Rf}$ described in (6) is a criteria for detection fault in power system. Fault resistance value calculated in the normal lines is very high (Mohm) like open circuit. If the healthy line is in normal operating mode encounter virtual fault (Load Encroachment), then Rf value will be still high which indicating that no fault occurred. Hence, it can be prevented from sending the open command. However, due to extreme fluctuations in load, there is little change in voltage or current but fault resistance value is still very high. If a real fault occurs, the calculated $\mathrm{Rf}$ will decrease considerably indicating real short circuit occurrence.

It should be noted that the operation time of Backup protection (Third zone), considering communication delay time is approximately, $600 \mathrm{~ms}$ which is in the range of typical third zone of distance protection. Therefore, the third zone backup protection can be replaced with proposed wide area protection system.

\section{Simulation Results}

As discussed in the first Section, lack of a proper solution to load encroachment has led some regions in the US and Canada to completely eliminate the third zone on $230 \mathrm{kV}$ lines and above [22]. In this paper, the proposed structure has been tested on the IEEE 9 bus Test (WSCC) system [23]. The simulation been carried out by using the MATLAB Simulink software. Fig (5) shows the IEEE 9 bus test system. the values shown are in per unit at $60 \mathrm{~Hz}$ on a 100MVA base [23].

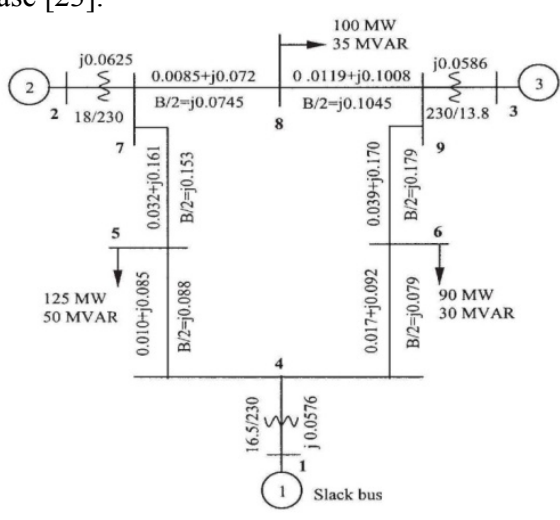

Fig5. Three Machines 9-Bus IEEE Standard Network (WSCC).
All Iine order to implement the structure, phasor measurement Unit (PMU) simulated on all bus network for accurate measurement of voltage and current and implementation. Fig (6) shows the IEEE 9 bus Test (WSCC) system simulation.

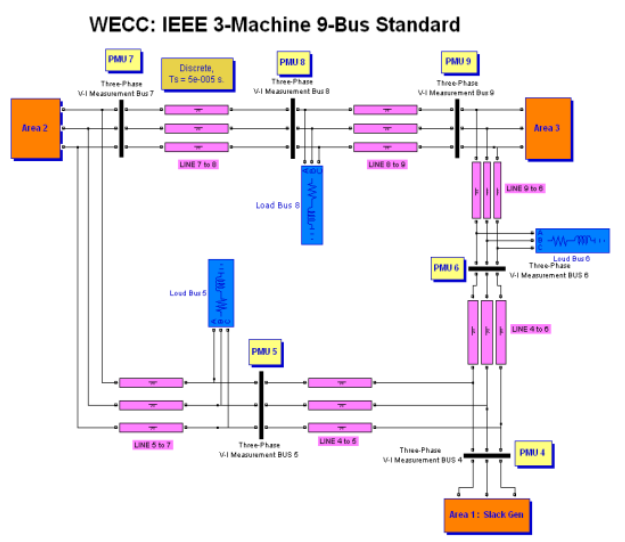

Fig6. Shows the IEEE 9 bus Test (WSCC) system simulation

In phasor measurement simulation, the sample measurement of voltage and current inputs are collected by the $\mathrm{A} / \mathrm{D}$ (Analog to digital converter) at the rate of 48 samples/cycle. The sampling interval is controlled by a well-proven frequency-tracking algorithm in order to respond dynamically to changes in system frequency. Fig (7) below shows the phasor measurement unit (PMU) simulation.

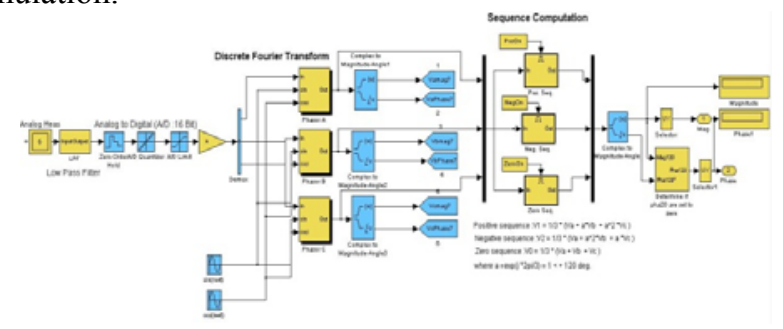

Fig 7. Phasor Measurement Unit (PMU) Simulation

Scenario 1. Simulation of the Third Zone Distance Relay For better understanding, using data of phasor measurement Unit (PMU), the third zone of distance relay on the classical term be simulated and its performance against the Load Encroachment is reviewed. Most digital impedance relays offer load encroachment functions to account for heavily loaded conditions. The North America Electric Reliability Council (NERC) has recommended using a $150 \%$ thermal rating with a $0.85 \mathrm{pu}$ voltage at power factor angle of 30 degrees [3]. The magnitudes of these parameters were determined to be observed under extreme conditions but not in a cascading mode. In this scenario of simulation, it can be seen that the measured value of impedance in Load Encroachment can be entered into the third zone of relay and will be causing unwanted outages. Distance relay placed on bus (7) and Load Encroachment in Line Bus7 to Bus 9 of network has been created and can be seen behavior of third zone in Fig (8). 


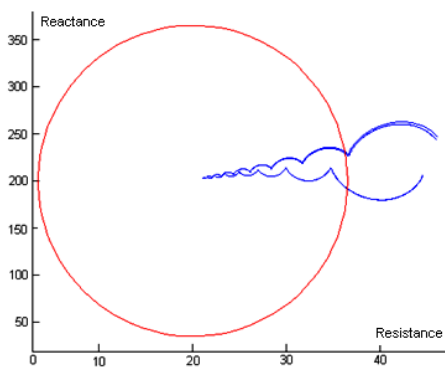

Fig 8. Trajectory of Impedance third zone during Load Encroachment

As you can see in Fig (9), after the setting time of third zone, due to impedance path enter the third zone, close command of CB is removed and the Relative CB will be opened and cause to overload other line.

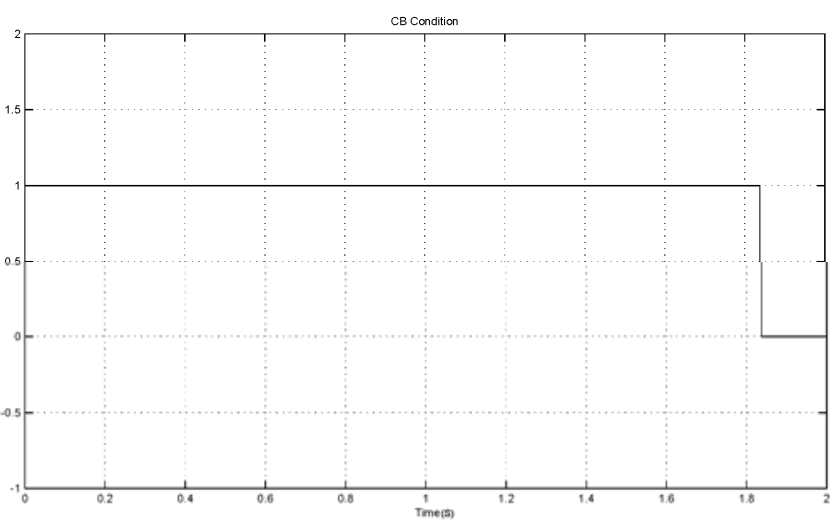

Fig9. Open CB Command when Load Encroachment occurred

Scenario 2.Simulation of the Proposed Structure during Normal Condition

All calculations in this scenario based on parameter (Rf) are performed and can be seen in Fig (10). Given very high resistance and lack of any fault, the network continues to operation normally, which indicates the validity of the proposed structure and parameter. Very high value of parameter (Rf) indicates no fault in the line of network. In this scenario, to simplify, As can be seen in fig (10), the value of (Rf) is very high (Mohm).

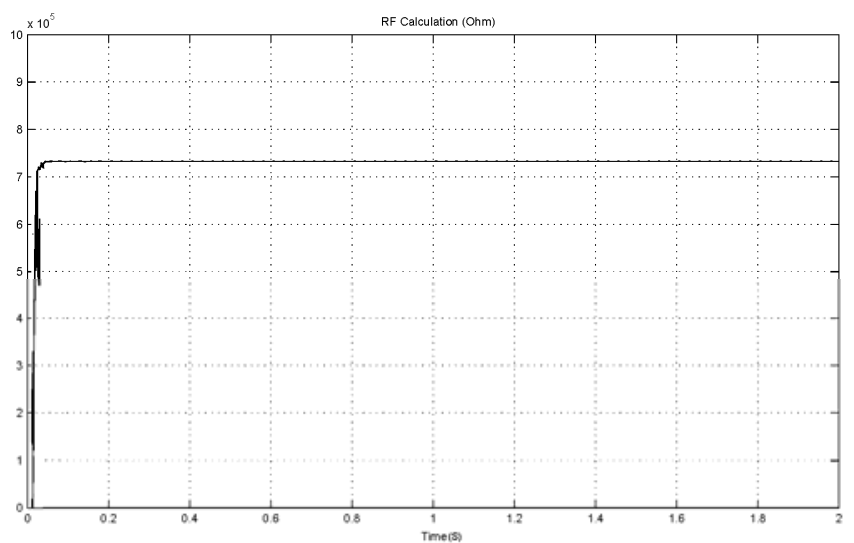

Fig 10. The value of (Rf) (approximate1Mohm) in normal operation

Scenario 3. Simulations of the Proposed Structure during Short Circuits

In this scenario, the performance of parameter (Rf) will be reviewed in real fault like short circuit. A real fault (short circuit) in the protection area of third zone considered and value of parameter will be assessed. An actual fault such as three phase short circuit between lines bus 7 to bus 9 is be considered. In Fig (11) can be seen that the value of $(\mathrm{Rf})$ after real fault will be decreased to approximately zero and after the setting time, open command is send to related $\mathrm{CB}$.

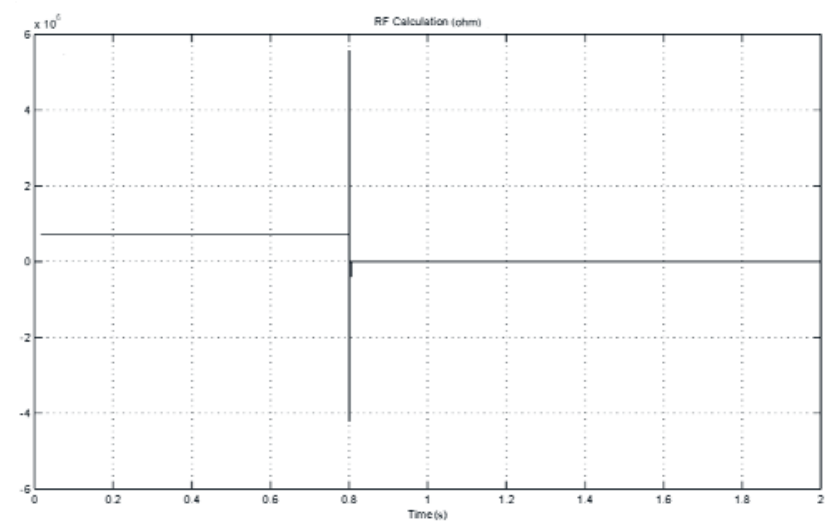

Fig11. The value of (Rf) (zero Mohm) in real fault (short circuit)

As can be seen in Fig (12), the short circuit as soon as occurred, fault detection and calculations of backup protection in a very short period (less than 200 milliseconds) is done. Fig (13) shows that Computation time of backup protection, sending and receiving is less than 600 milliseconds, which is near conventional protective systems.

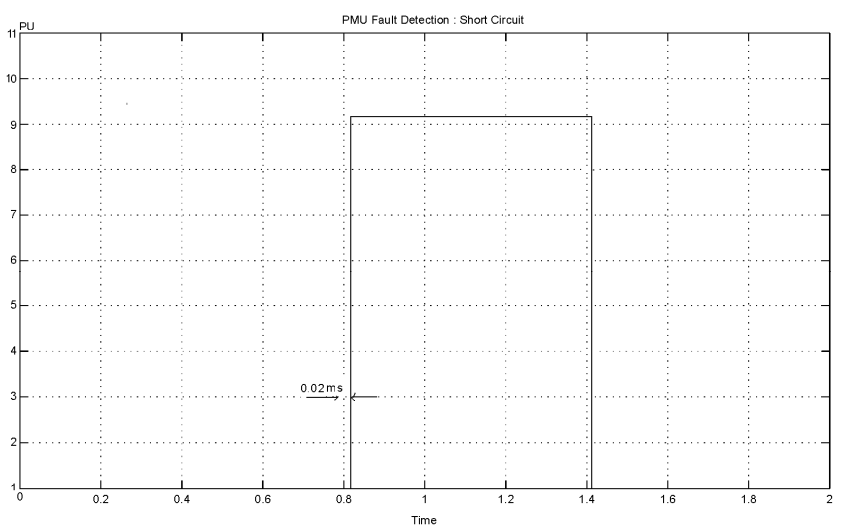

Fig12. Short Circuit Current Detection

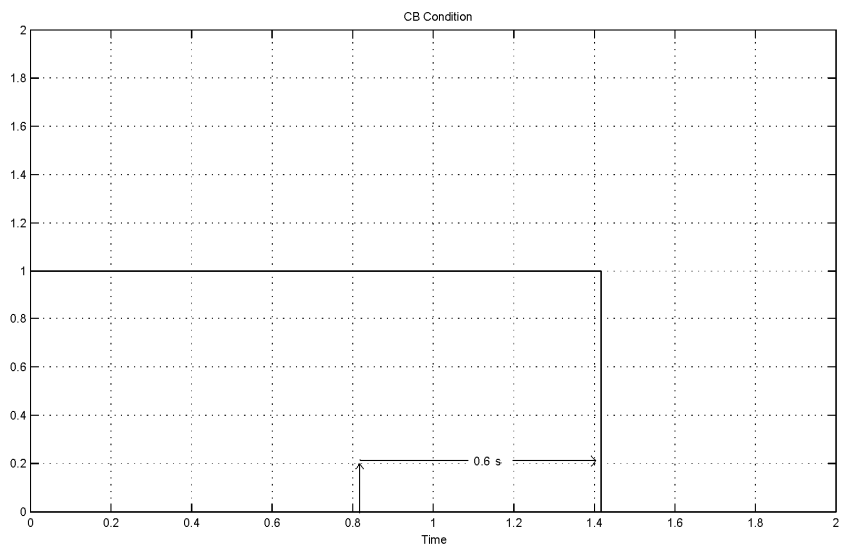

Fig13. Calculation and send Open CB Command in $600 \mathrm{~ms}$ 
Scenario 4. Simulation of the Proposed Structure during Abnormal Operation

In this scenario, the proposed method is investigated during an abnormal operation such as load Encroachment. To achieve these conditions, Load of bus 8 is changed such that the impedance come into protection zones. The value of parameter (Rf) shown in Fig (14) is high (less than normal) that indicated no fault in system.

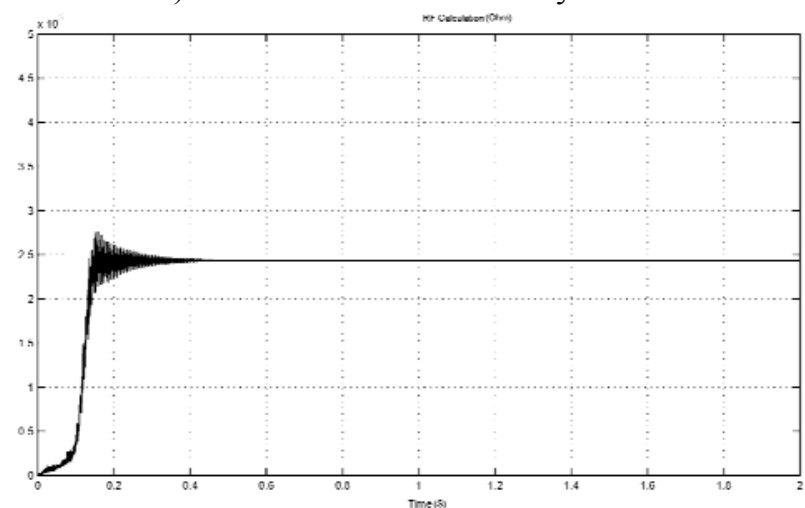

Fig14. The value of (Rf) (approximate $0.2 \mathrm{Mohm}$ ) in abnormal operation

\section{Conclusions}

The survey of simulation results for different scenarios has been done in all three operating modes, normal condition, abnormal condition (load encroachment) and a real fault. the result have shown that the proposed structure is effective for different network conditions as backup protection (third zone). According to simulation results, the fiber optics as a communication platform has latency of approximately 300 milliseconds round-trip path $(100-150 \mathrm{~km})$. Processing time needed for computation and simulation-based decision on proposal is about 200 milliseconds. The total time value of wide-area backup protection system (WABPS) less than 600 milliseconds that is near current third zone $(600 \mathrm{~ms}-1500 \mathrm{~ms})$. The decrease time of this proposed structure beside the current backup protection (600 to 1500 milliseconds) cause a faster diagnosis of abnormal conditions or fault condition and increase margin of power system stability and reliability.

\section{References}

[1]. Terzija Vladimir, Valverde Gustavo, Cai Deyu, Regulski Pawel, Madani Vahid, Fitch John, SkokSrdjan, Miroslav M.Begovic \& Phadeke A "Wide-Are Monitoring,Protection, and Control of Future Electric Power Networks", Presented At Proceedings of the IEEE, VOL. 99, NO. 1, Jan 2011, PP.80-93.

[2]. Machowski J, W Bialek J, R Bumby J," Power System Dynamics: Stability \& Control", 2nd Edition 2008, Press John Willy \& Sons, Ltd .

[3]. U.S.-Canada Power System Outage Task Force, "Final Report on the August 14, 2003 Blackout in the United States and Canada Causes and Recommendations" April 2004.

[4]. Yuchen Lu, Holbach Juergen, Martuscello Laurie, Krizauskas Edward," Tests of Distance Relay Performance on Stable and Unstable Power Swings Using
Simulated Data of the August 14th 2003 System Disturbance" Presented at DistribuTech/TransTech San Diego, California Feb. 4, 2009

[5]. Kundur Prabha," Power System Stability and Control", March 1994, Press McGraw-Hill Inc ,Chapter 2 $\& 14$.

[6]. Grenor Verbic, Ferdinand Gubina, "A New Concept of Voltage-Collapse Protection Based on Local Phasors", IEEE Transactions on Power Delivery, Vol.1 9 No.2, Apr. 2006, PP.576-581.

[7]. Jonsson Mattias, E. Daalder Jaap, “An Adaptive Scheme to Prevent Undesirable Distance Protection Operation During Voltage Instability" IEEE Transactions on Power Systems Delivery, VOL. 18, NO. 4,Oct 2003, PP.1174-1180.

[8]. Bin Abidin Ahmad Farid, Mohamed Azah, "Adaptive Settings of Distance Protection to Prevent Mal-operation under Severe Voltage Fluctuation" IEEE International Conference on System Engineering and Technology 2011 (ICSET),PP162-165.

[9]. Jemal Yunus Kalid, Pinares Gustavo, Tuan Le Anh, Bertling Lina ," A Combined Zone-3 Relay Blocking and Sensitivity-Based Load Shedding for Voltage Collapse Prevention", Innovative Smart Grid Technologies Conference Europe (ISGT Europe), 2010 IEEE PES11-13 Oct. 2010 PP: $1-8$.

[10]. Seong-Il Lim, Chen-Ching Liu, Seung-Jae Lee, Myeon-Song Choi, \& Seong-Jeong Rim, "Blocking of Zone 3 Relays to Prevent Cascaded Events" IEEE Transactions on Power Systems, VOL. 23, NO. 2, May 2008, PP.747-754.

[11]. Gang Shen, Venkataramana Ajjarapu, A Novel Algorithm Incorporating System Status to Prevent Undesirable Protection Operation during Voltage Instability, 39th North American Power Symposium, 2007, NAPS'07, Sept.3 0 2007-0ct. 2 2007, PP. 373 - 378. [12]. Seong-Il Lim, Chen-Ching Liu, Seung-Jae Lee, Myeon-Song Choi, \& Seong-Jeong Rim, "Blocking of Zone 3 Relays to Prevent Cascaded Events" IEEE Transactions on Power Systems, VOL. 23, NO. 2, May 2008, PP.747-754

[13]. Xiangning Lin, Hanli Weng, Bin Wang, Zhiqian Bo,".A Fault-Component based Adaptive Distance Protection Schame" Power \& Energy Society General Meeting, July 2009. PP.26-30

[14]. Ching-Shan Chen, Chih-Wen Liu, and Joe-Air Jiang, "A New Adaptive PMU Based Protection Scheme for Transposed/Untransposed Parallel Transmission Lines", IEEE Transactions on Power Systems, VOL. 17, NO. 2, April 2002

[15].Shravan Garlapati, Hua Lin, Santhoshkumar Sambamoorthy, Sandeep K. Shukla, James Thorp, “Agent Based Supervision of Zone 3 Relays to Prevent Hidden Failure Based Tripping", International Conference on Smart Grid Communications 2010, PP.256 - 261

[16].D.Tholomier, HKang, BCvorovic,'Phasor Measurements Functionality and Applications", Power Systems Conference 2009, Clemson University Advanced Metering,Protection, Control, Communication and Distributed Resources 
[17]. Phadke A.G, Thorp J.S,'History and Application of Phasor Measurements", Power Systems Conference and Exposition, PSCE 2006, PP.331-335

[18].E.Martin Kenneth, "Synchrophasor Standards development IEEE C37.118 \& IEC 61850”, Proceedings of the 44th Hawaii International Conference on System Sciences 2011.

[19]. S. Tanenbaum Andrew, "Computer Networks (4th Edition)", Press Prentice Hall; 4 Edition (August 19, 2002).

[20]. Naduvathuparambil Biju, Valenti, Matthew C, Feliachi Ali ,"Communication Delays in Wide Area Measurement Systems", Presented At Proceedings of the Thirty-Fourth Southeastern Symposium on System Theory 2002,PP.118-122.

[21]. Amr El-Hadidy \& Christian Rehtanz," A New Algorithm to Improve the Operation of Distance Relays Zone 3 by Using Synchronized Phasor Measurements", Modern Electric Power Systems 2010, Wroclaw, Poland 2010.

[22]. Andersson, P. Donalek, R. Farmer, N. Hatziargyriou, I. Kamwa, P. Kundur, N. Martins, J. Paserba, P. Pourbeik, J. Sanchez-Gasca, R. Schulz, A. Stankovic, C. Taylor, and V. Vittal, "Causes of the 2003 Major Grid Blackouts in North America and Europe, and Recommended Means to Improve System Dynamic Performance" IEEE Transactions on Power Systems, VOL. 20, NO. 4, Nov 2005, (PP.1922-1928)

[23]. P. W. Sauer and M. A. Pai. Power System Dynamics and Stability.Prentice-Hall, Upper Saddle River, NJ, 1998 